Korean Chem. Eng. Res., 52(3), 340-346 (2014)

http://dx.doi.org/10.9713/kcer.2014.52.3.340

PISSN 0304-128X, EISSN 2233-9558

\title{
Preparation and Gas Permeability of ZIF-7 Membranes Prepared via Two-step Crystallization Technique
}

\author{
Fang Li, Qiming Li ${ }^{\dagger}$, Xinxia Bao, Jianzhou Gui and Xiaofei Yu \\ College of Chemistry, Chemical Engineering and Environmental Engineering, Liaoning Shihua University, \\ Fushun Liaoning 113001, P.R. China \\ (Received 19 December 2013; Received in revised form 14 February 2014; accepted 20 February 2014)
}

\begin{abstract}
Continuous and dense ZIF-7 membranes were successfully synthesized on $\alpha-\mathrm{Al}_{2} \mathrm{O}_{3}$ porous substrate via two-step crystallization technique. ZIF-7 seeding layer was first deposited on porous $\alpha-\mathrm{Al}_{2} \mathrm{O}_{3}$ substrate by in-situ low temperature crystallization, and then ZIF-7 membrane layer can be grown through the secondary high-temperature crystallization. Two synthesis solutions with different concentration were used to prepare ZIF-7 seeding layer and membrane layer on porous $\alpha-\mathrm{Al}_{2} \mathrm{O}_{3}$ substrate, respectively. As a result, a continuous and defect-free ZIF-7 membrane layer can be prepared on porous $\alpha-\mathrm{Al}_{2} \mathrm{O}_{3}$ substrate, as confirmed by scanning electron microscope. XRD characterization shows that the resulting membrane layer is composed of pure ZIF-7 phase without any impurity. A single gas permeation test of $\mathrm{H}_{2}, \mathrm{O}_{2}, \mathrm{CH}_{4}$ or $\mathrm{CO}_{2}$ was conducted based on our prepared ZIF-7 membrane. The ZIF-7 membrane exhibited excellent $\mathrm{H}_{2}$ molecular sieving properties due to its suitable pore aperture and defect-free membrane layer.
\end{abstract}

Key words: ZIF-7 Membrane, Two-step Crystallization, Precursor Solution, Gas Permeation

\section{Introduction}

Metal-organic frameworks (MOFs) with zeolite-like structural and chemical properties are an emerging class of crystalline hybrid materials [1-5]. MOFs have attracted more and more attention due to their potential application in gas adsorption, gas separation, catalysis, drug-delivery or chemical sensors based on their ultra-high specific surface area and pore volume [6-9]. In MOFs, the metal clusters, dubbed secondary building units (SBUs), are bound together via multifunctional organic linkers to form one-, two- or three-dimensional networks. After removing the trapped guest molecules, a robust and porous crystalline material can be formed. MOFs can extend extremely high accessible porosity by the same order of magnitude or even higher compared with other zeolitic or mesoporous materials [10]. So far more than 180 MOF-type materials with diverse framework architectures and functional properties have been developed based on a diversity of organic linkers and metal clusters. Amongst zeolitic imidazolate frameworks (ZIFs), a subfamily of MOFs, have emerged as a novel type of porous crystalline material which combines excellent properties from zeolite and MOFs [1113]. For instance, the topographical structure of ZIFs materials resembles that of zeolite in which metal clusters are linked by imidazolate-related organic linkers. Hence, a variety of ZIFs with high surface area, exceptional chemical and thermal stability have been synthesized, e.g., ZIF-8 exhibits high specific area of up to $1810 \mathrm{~m}^{2} / \mathrm{g}$

\footnotetext{
${ }^{\dagger}$ To whom correspondence should be addressed.

E-mail: lqm dicp@163.com

This is an Open-Access article distributed under the terms of the Creative Commons Attribution Non-Commercial License (http://creativecommons.org/licenses/by$\mathrm{nc} / 3.0$ ) which permits unrestricted non-commercial use, distribution, and reproduction in any medium, provided the original work is properly cited.
}

and high thermal stability of up to $550^{\circ} \mathrm{C}[14]$. Hence, ZIFs possess excellent prospects in many areas $[15,16]$.

Developing energy-efficient separation processes is an important research topic in dealing with greenhouse gases $\left(\mathrm{CO}_{2}\right)$, hydrogen or toxic gases etc. Compared to conventional separation, the geometrical configuration of supported membranes possesses significant advantage in gas separation application [21]. Therefore, the versatility in pore aperture and specific surface area enables ZIF-based membranes to be very promising for separating small-sized gas mixtures. For example, the pore size of ZIF-8 is $c a .0 .34 \mathrm{~nm}$, which is more suitable for separating $\mathrm{H}_{2}(0.29 \mathrm{~nm}), \mathrm{CO}_{2}(0.33 \mathrm{~nm})$ and $\mathrm{CH}_{4}(0.38 \mathrm{~nm})$ than some zeolite membranes (LTA $(0.41 \mathrm{~nm}), \operatorname{MFI}(0.53 \sim 0.56 \mathrm{~nm})$ [17-20]. There have been many attempts to prepare ZIFs-related membranes in the past several years. Particularly, some ZIF-based membranes have been successfully fabricated and demonstrated molecular sieving properties. Venna et al. synthesized ZIF-8 membrane layer on porous $\alpha$-alumina tube using secondary seeded growth method which displays $\mathrm{CO}_{2}$ permeance as high as $\sim 2.4 \times 10^{-5} \mathrm{~mol} / \mathrm{m}^{2} \cdot \mathrm{s}$. Pa and $\mathrm{CO}_{2} / \mathrm{CH}_{4}$ separation selectivity of $4 \sim 7$ [22]. Bux et al. prepared ZIF-8 membranes with in-situ microwave-assisted solvothermal method, and they found that the ZIF-8 membrane demonstrated an optimized $\mathrm{H}_{2}$ selectivity with respect to other gases [23,24]. Huang et al. suggested that 3-Aminopropyltriethoxysilane (APTES) can help to form defect-free ZIFs membranes and thus developed ZIF-22 membranes with APTES as covalent linker. Liu et al. successfully prepared ZIF-69 membrane on porous $\alpha$-alumna support by in-situ solvothermal method and they found that the $\mathrm{CO}_{2} / \mathrm{CO}$ permselectivity of $3.5 \pm 0.1$ and $\mathrm{CO}_{2}$ permeance of $3.6 \pm 0.3 \times 10^{-8} \mathrm{~mol} \cdot \mathrm{m}^{-2} \cdot \mathrm{s}^{-1}$. $\mathrm{Pa}^{-1}$ at room temperature can be obtained [25]. ZIF-7 membranes were also prepared using microwave-assisted method, and this membrane 
exhibits a promising $\mathrm{H}_{2}$ separation performance. Generally, the organicinorganic hybrid ZIFs have a more flexible framework structure compared with the rigid zeolite, e.g., MIL-53 exhibits a very large breathing effect [26]. Flexible framework of ZIFs enables some gases to permeate the pores of ZIFs with smaller size, which impairs the molecular-sieving performance. In this regard, ZIF-7 with smaller pore size (ca. $0.33 \mathrm{~nm}$ ) should find more promising potential in small molecular gas separation compared with ZIF-8 (ca. $0.34 \mathrm{~nm}$ ) [28]. As we know, most ZIF-7 membranes were synthesized through secondary seeded growth method [27]. Especially, the dip-coating method is always used to deposit ZIF-7 seeding layer on porous substrate, but with this method it is difficult to maintain high reproducibility due to human manipulating factor. Hence, a simple, effective and highly repetitive seeding coating method needs to be developed for preparing ZIF-7 membranes on porous substrate.

Our aim was to use in-situ crystallization procedure to replace traditional dip-coating seeding method and simplify the seeding process. Herein, two crystallization procedures were adopted to prepare ZIF-7 membranes: one crystallization procedure was used to deposit ZIF-7 seeding layer at room temperature, and the other was used to grow ZIF-7 membrane at high temperature. In this article, we present the synthesis, characterization, and separation performance of asprepared ZIF-7 membrane.

\section{Experimental}

\section{2-1. Chemical materials}

Zinc nitrate hexahydrate $\left(\mathrm{Zn}\left(\mathrm{NO}_{3}\right)_{2} .6 \mathrm{H}_{2} \mathrm{O}, 96 \%\right)$ was purchased from Junsei Chemical Co., Ltd. and benzimidazole (98\%) was purchased from Aldrich Chemical Co., Ltd. All chemicals were used as supplied without further purification. N,N-dimethylformamide (SHOWA chemical Co., Ltd., 99.5\%) was used as a solvent in solvothermal synthesis.

\section{2-2. Preparation of the substrate}

The round $\alpha$-alumina porous substrate for supporting ZIF-7 membrane was homemade by an isostatic pressing method. Typically, high purity alumina powders $\left(\mathrm{Al}_{2} \mathrm{O}_{3}\right)$ were first pressed into green discs with a thickness of $1.5 \mathrm{~mm}$ and a diameter of $25 \mathrm{~mm}$ with the aid of a hydraulic press (Carver), and then sintered at $1380{ }^{\circ} \mathrm{C}$ for $40 \mathrm{~h}$. Next, the substrates were sonicated for $10 \mathrm{~min}$ in DI water to remove the loosed alumina particles and for $10 \mathrm{~min}$ in ethanol to eliminate organic impurity. To further reduce the surface macropore defects, an alumina sol was spin-coated onto the top surface of the porous substrate. Afterwards it was again sintered at $500{ }^{\circ} \mathrm{C}$ for reinforcing alumina sol. Finally, the substrates were washed using DI water and ethanol solution, respectively. After drying at $100^{\circ} \mathrm{C}$ for $6 \mathrm{~h}$, the porous $\alpha$-alumina discs could be used as the substrates.

\section{2-3. Preparation of ZIF-7 membranes}

In the experiment, a combined room-temperature crystallization and high- temperature crystallization were used to grow ZIF-7 membrane layer on the $\alpha$-alumina substrate. Here two precursor solutions with different concentrations were used in respective crystallization processes. $0.25 \mathrm{~g}$ benzimidazole and $0.05 \mathrm{~g}$ zinc nitrate hexahydrate were dissolved into $60 \mathrm{ml}$ DMF solvent. After $30 \mathrm{~min}$ of vigorous stirring a clear homogeneous solution can be obtained. The pretreated substrate was put into the Teflon-lined steel autoclave vertically with a Teflon holder, avoiding crystal sedimentation. Then the resultant synthesis solution was transferred into the autoclave. The autoclave was maintained at room temperature for $24 \mathrm{~h}$. Afterwards, the disk was taken out and dried at $80{ }^{\circ} \mathrm{C}$ for $12 \mathrm{~h}$. Secondary synthesis solution was prepared by mixing $0.6 \mathrm{~g}$ benzimidazole and $1.14 \mathrm{~g}$ zinc nitrate hexahydrate into $60 \mathrm{ml}$ DMF. Then the solution was poured into the autoclave. The autoclave was heated under autogenous pressure in a conventional oven at $130^{\circ} \mathrm{C}$ for $24 \mathrm{~h}$. After synthesis the membrane samples were taken out, rinsed frequently by methanol, and then with chloroform and methanol for three times. After drying at $50{ }^{\circ} \mathrm{C}$ overnight, the membrane can be used for characterization and testing the gas permeation properties. For comparison, another membrane sample was also prepared using the synthesis solution (dissolving $0.6 \mathrm{~g}$ benzimidazole and $1.14 \mathrm{~g}$ zinc nitrate hexahydrate into $60 \mathrm{ml} \mathrm{DMF}$ ), but the membrane sample was obtained only through single-step crystallization $\left(130^{\circ} \mathrm{C}\right.$ for $\left.24 \mathrm{~h}\right)$. The posttreatment method was similar to the ZIF-7 membrane prepared by two-step crystallization.

\section{2-4. Characterization of the samples}

The surface morphologies and cross-section of ZIF-7 membranes were observed with SEM (Hitachi, S-3500N). The phase structure of ZIF-7 membrane layer was identified with X-ray diffractometer in the $2 \theta$ range of $5 \sim 50^{\circ}$ (PANalytical, X'pert-Pro). Thermal gravimetric analysis (TA Instrument, TGA2950) was performed on ZIF-7 powder samples to investigate their thermal stability. FTIR was conducted on Varian 2000 Fourier infrared spectrometer to investigate the structural information of ZIF-7 powders from the crystallization.

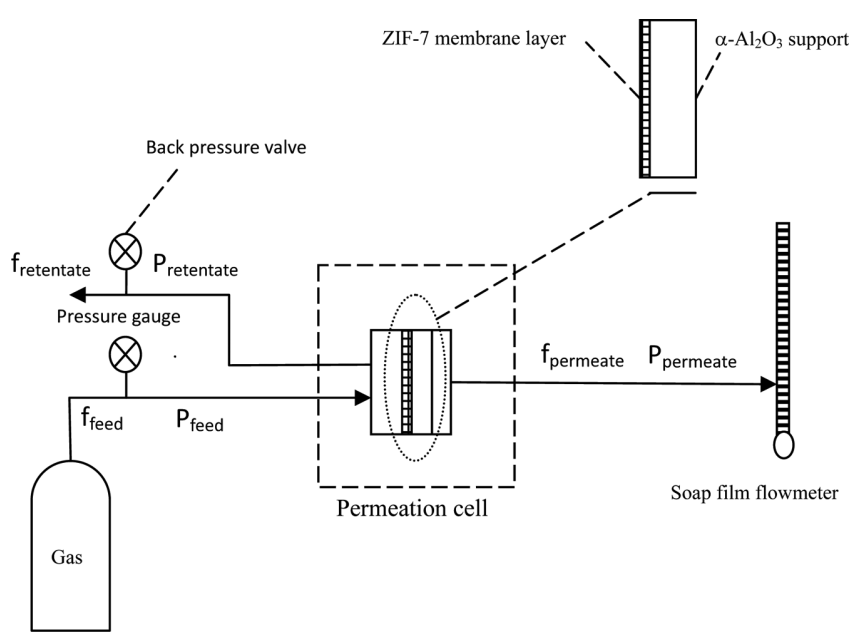

Fig. 1. The homemade setup for single gas permeation. 


\section{2-5. Gas permeation of ZIF-7 membranes}

Single gas permeability through ZIF-7 membranes was checked on our homemade apparatus (Fig. 1). The permeance and ideal selectivity of small gas molecules $\left(\mathrm{H}_{2}, \mathrm{CH}_{4}, \mathrm{~N}_{2}\right.$, and $\left.\mathrm{CO}_{2}\right)$ through ZIF-7 membrane or $\alpha$-alumina substrates were determined at room temperature. The feed-side pressure was adjusted by a reducing valve and back pressure valve and the permeate side was kept constant at normal atmosphere. At every permeation process, the measurement system was stabilized for at least $2 \mathrm{~h}$ and the measurement was repeated three times. Thus the data could be obtained by averaging the permeation data.

\section{Results and Discussion}

\section{3-1. XRD, TG, FTIR characterization of ZIF-7 membranes}

Generally during the pre-coated seeding process, ZIF-7 seeding particles should be synthesized in advance and then coated onto porous substrate by a dip-coating method. To simplify the aforementioned procedure, the seeded $\alpha-\mathrm{Al}_{2} \mathrm{O}_{3}$ substrate was obtained through in-situ crystallization at room temperature. As we know, excessive benzimidazole to zinc nitrate in synthesis solution can produce nanosized ZIF-7 particles. These ZIF-7 nanoparticles can be easily sucked into the pores of porous substrate or formed directly in the substrate. As a result, the diffraction intensity of ZIF-7 seeding layer was weak. During the secondary growth process, the seeded substrate is exposed to the more concentrated synthesis solution as mentioned in the experimental section. Then a continuous and dense ZIF-7 membrane layer can be formed on porous substrate.

$X$-ray diffraction measurement was performed to identify the phase structure of ZIF-7 membrane and porous substrate. Fig. 2 shows the XRD patterns of $\alpha-\mathrm{Al}_{2} \mathrm{O}_{3}$ porous substrate, seeded porous substrate

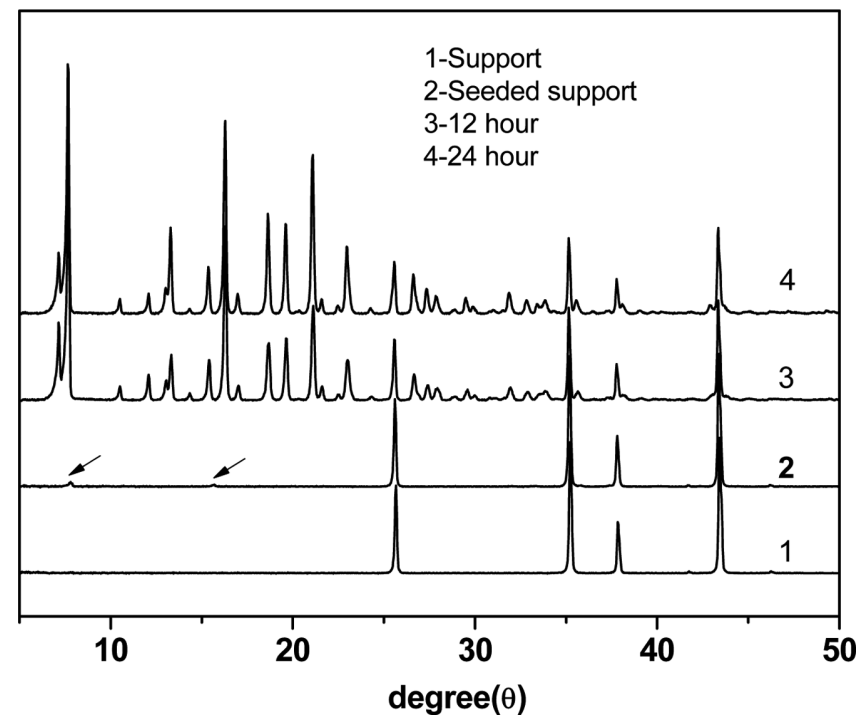

Fig. 2. XRD patterns of $\alpha$-alumina substrate, seeded substrate and ZIF-7 membrane from two-step crystallization. 1-Pure $\alpha$ alumina substrate; 2-The seeded substrate; 3-ZIF-7 membrane layer crystallized for 12hours; 4-ZIF-7 membrane layer crystallized for 24 hours. and ZIF-7 membrane layer. As shown in Fig. 2-1 and Fig. 2-2, there are subtle differences between $\alpha-\mathrm{Al}_{2} \mathrm{O}_{3}$ substrate and the seeded substrate, e.g., the seeded substrate exhibits some tiny characteristic peaks at $7.80^{\circ}$ and $15.98^{\circ}$ which can account for the presence of ZIF7 nanocrystals. The distinct XRD patterns for ZIF-7 membrane can be obtained after $12 \mathrm{~h}$ (Fig. 2,3). And XRD patterns of the ZIF-7 membranes are well matched with the reported ZIF-7 powders, which exhibit the typical characteristic peaks of ZIF-7 at $2 \mathrm{q}=7.15^{\circ}$, $7.65^{\circ}, 10.51^{\circ}, 12.09^{\circ}, 13.03^{\circ}, 15.37^{\circ}, 18.64^{\circ}, 19.60^{\circ}$ and $21.10^{\circ}$ [29]. While the crystallization time is prolonged to $24 \mathrm{hrs}$, the peak intensity of ZIF-7 membrane further increases (Fig. 2 4). It can be concluded that high-temperature crystallization $\left(130^{\circ} \mathrm{C}\right)$ can accelerate the crystallization rate and improve membrane integrity. In addition, four diffraction peaks at $25.7^{\circ}, 35.0^{\circ}, 38.02^{\circ}$ and $43.33^{\circ}$ in Fig. $2-4$ are ascribed to $\alpha-\mathrm{Al}_{2} \mathrm{O}_{3}$ substrate. However, their diffraction intensity is much weaker than that of pure $\alpha-\mathrm{Al}_{2} \mathrm{O}_{3}$ substrate due to the formation of the continuous ZIF-7 membrane layer.

After secondary growth, the residual ZIF-7 powders were collected from the autoclave bottom and characterized with TG and FTIR. The thermal stability of the as-synthesized ZIF-7 crystals is presented in Fig. 3. There are two important weight losses from room temperature to $850^{\circ} \mathrm{C}$ : approximately $5.0 \%$ weight loss occurs at $150 \sim$ $250{ }^{\circ} \mathrm{C}$, resulting from the removal of DMF solvent molecules from the framework. The removal of those trapped guest molecules can give rise to huge specific surface area for ZIF-7. Another important weight loss, in line with around $50.0 \%$, occurs between $500{ }^{\circ} \mathrm{C}$ and $600{ }^{\circ} \mathrm{C}$, indicating the thermal decomposition of ZIF-7. In this process, most organic components of ZIF-7 can be pyrolyzed and only inorganic components such as $\mathrm{ZnO}$ may be left. TG analysis proves that our synthesized ZIF-7 membrane is thermally stable up to nearly $500{ }^{\circ} \mathrm{C}$.

FT-IR was also conducted to check the organic groups of ZIF-7 powders from the autoclave bottom. Fig. 4 gives FT-IR spectra of benzimidazole, zinc nitrate and ZIF-7 crystals, respectively. The C$\mathrm{H}$ stretch vibration of benzimidazole occurs near $3100 \sim 3000 \mathrm{~cm}^{-1}$.

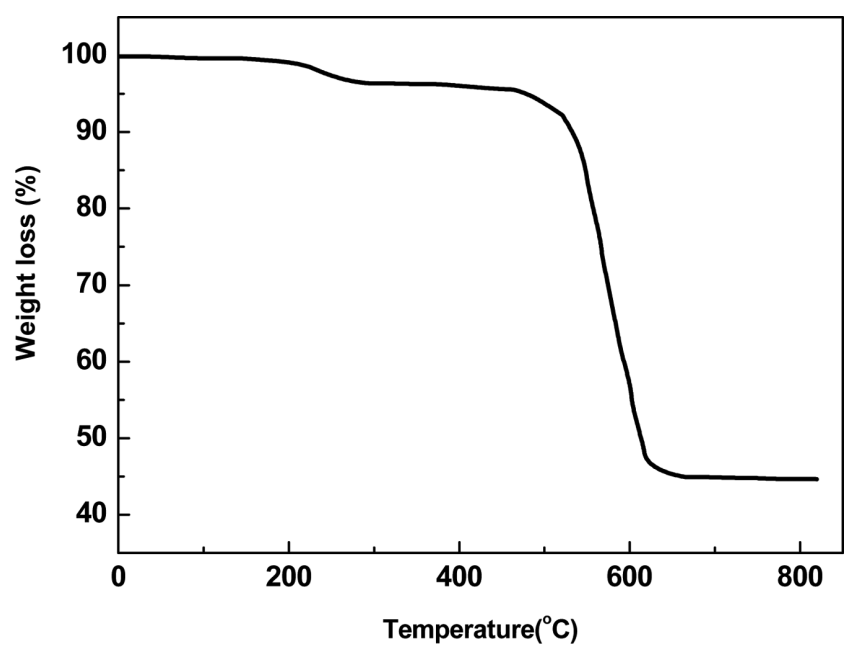

Fig. 3. TG curve of the ZIF-7 powders from the autoclave bottom. 


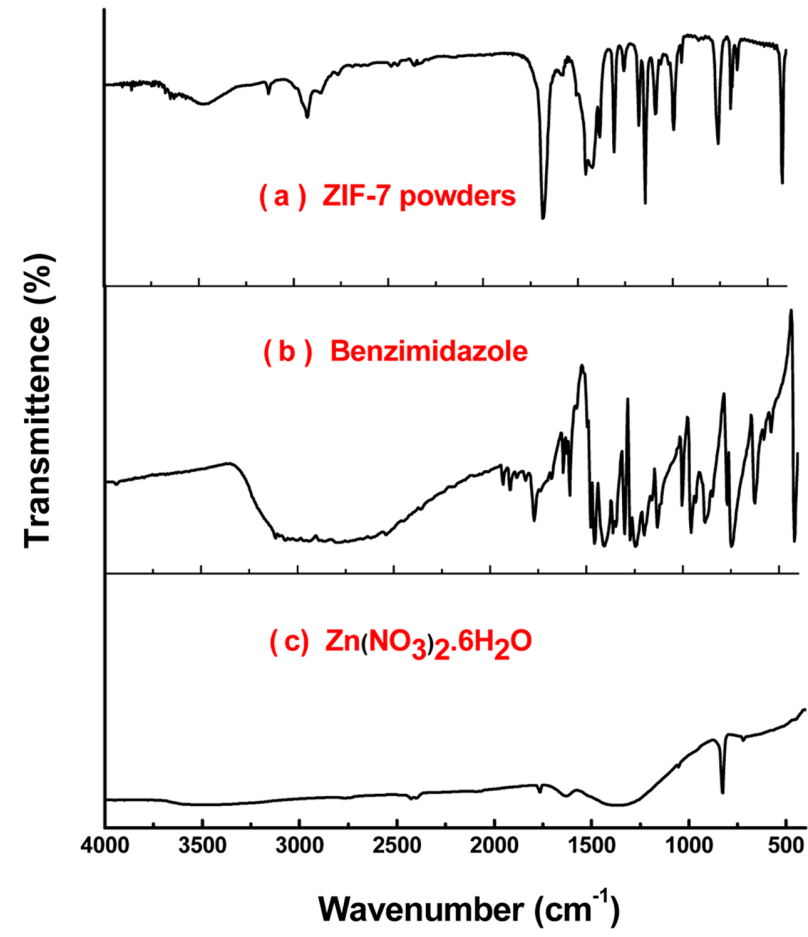

Fig. 4. FTIR spectra of ZIF-7 powder from the autoclave bottom. (a) denotes ZIF-7powders; (b) denotes benzimidazole; (c) denotes zinc nitrate hexahydrate.

The $\mathrm{C}=\mathrm{C}$ stretch band of aromatic ring of benzimidazole absorbs at about $1600 \sim 1500 \mathrm{~cm}^{-1}$. For benzimidazole, the stretch band of N-H group absorbs strongly at $3500 \sim 2500 \mathrm{~cm}^{-1}$, which is accompanied by bend vibration of N-H group near $1650 \sim 1580 \mathrm{~cm}^{-1}$. As shown in Fig. 4, the disappearance of strong and broad N-H band between $3250 \sim 2500 \mathrm{~cm}^{-1}$ means the deprotonation and the formation of ZIF-7. Correspondingly, the medium bend vibration peaks of N-H group near $1650 \sim 1580 \mathrm{~cm}^{-1}$ are absent in ZIF-7 FTIR spectra. Hence, FT-IR characterization illustrates that benzimidazole linker has been fully deprotonated during the crystallization and ZIF-7 is successfully synthesized.

\section{3-2. Surface and Cross-Section Microstructure of ZIF-7 Membranes}

We used a scanning electron microscope to examine the microstructure of ZIF-7 membrane layer. Fig. 5 compares the microstructure of ZIF-7 membranes prepared with two different synthesis methods. SEM images of ZIF-7 membranes from two-step crystallization are given in Fig. 5(a) and (b). It can be seen clearly that a dense and continuous ZIF-7 membrane is formed onto $\alpha$-alumina porous substrate via two-step crystallization, which is similar to the reported experimental results [27]. The crystal size of ZIF-7 crystals is approximately $10 \mu \mathrm{m}$, and these crystals can agglomerate into a large group or clusters, composing a compact and continuous membrane layer without obvious defects. The ZIF-7 membrane from single-step crystallization is also presented in Fig. 5(c) and (d). As shown in Fig. 5(c), a continuous ZIF-7 membrane layer can also be formed which is seemingly similar to the membrane synthesized by two-step crystal-
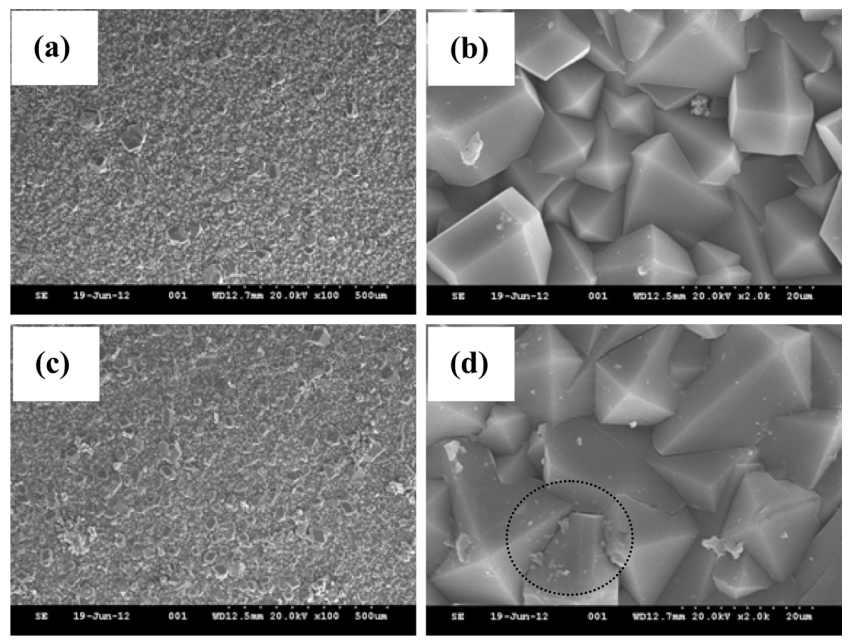

Fig. 5. The surface view of ZIF-7 membranes prepared by two-step crystallization and single-step crystallization; (a) and (b) represent the surface morphologies of ZIF-7 membrane from two-step crystallization; (c) and (d) represent the surface morphologies of ZIF-7 membrane using single step crystallization.
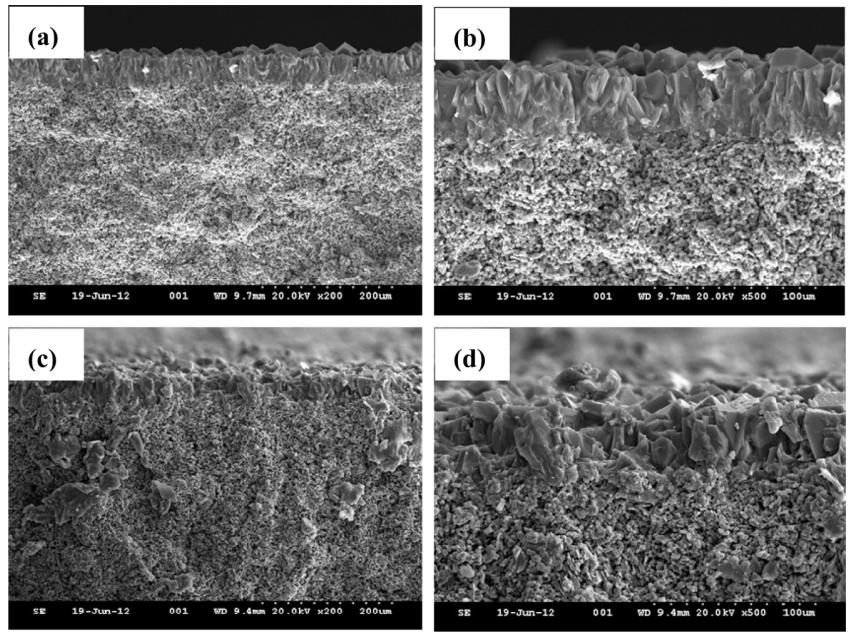

Fig. 6. The cross-section of the ZIF-7 membranes from two-step crystallization and single-step crystallization; (a) and (b) represent the cross-section of ZIF-7 membrane from two-step crystallization; (c) and (d) represent the cross-section of ZIF7 membrane from single step crystallization.

lization. However, the grain size of ZIF-7 membrane (single step crystallization) is larger than that from two-step crystallization as shown in Fig. 5(d). Consequently, the tiny defects existing in grain boundaries are inevitable for ZIF-7 membrane from single-step crystallization. In summary, two-step crystallization can produce better membrane samples with smaller crystal size and fewer defects.

Fig. 6 shows the cross-section view of ZIF-7 membrane prepared by two different synthetic methods. The cross-section microstructure of ZIF-7 membrane from two-step crystallization is shown in Fig. 6(a) and (b). It can be seen that a continuous and dense ZIF-7 membrane can be grown on $\alpha$-alumina porous substrate. The membrane thickness is very uniform, which is about $30 \mu \mathrm{m}$. The adhesion between porous substrate and ZIF-7 membrane layer is generally 


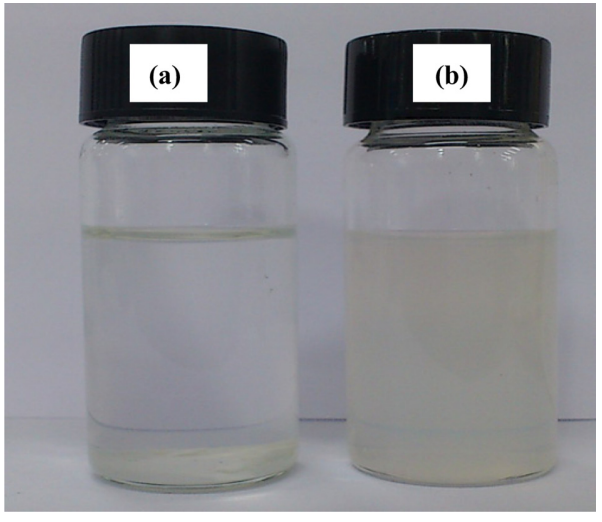

Fig. 7. Photos of the seeding synthetic solution; (a) denotes the low stoichiometric ratio of benzimidazole and zinc nitrate; (b) denotes the high stoichiometric ratio of benzimidazole and zinc nitrate.

weak due to different chemical properties. Here ZIF-7 seeding crystals can be firstly imbedded in a-alumina substrate via in-situ room temperature crystallization. Then strong attachment of ZIF-7 membrane layer onto porous substrate can be obtained through the secondary growth at high temperature.

According to some published papers, excessive benzimidazole in the synthetic solution can produce nanoscale ZIF-7 crystals [30-32]. In our experiment, $0.25 \mathrm{~g}$ benzimidazole and $0.05 \mathrm{~g}$ zinc nitrate hexahydrate are dissolved into $60 \mathrm{ml}$ DMF to prepare the seeding synthetic solution, where benzimidazole is much excessive. After 30 min of stirring at room temperature, a homogeneous opaque solution can be obtained (see Fig. 7(b)). It can be checked that ZIF-7 nanocrystals cannot deposit in DMF solvent for more than one week. For comparison, another synthetic solution was also prepared by mixing $0.25 \mathrm{~g}$ benzimidazole and $1.14 \mathrm{~g}$ zinc nitrate hexahydrate into $60 \mathrm{ml}$ DMF, where zinc nitrate is excessive. After $2 \mathrm{~h}$ of stirring at room temperature, white ZIF-7 crystals deposited at the container bottom and the transparent solvent can be clearly observed as shown in Fig. 7(a). The experimental result indicates that low stoichiometric ratio of benzimidazole to zinc nitrate can induce the formation of large ZIF-7 crystals. Herein with the aid of high stoichiometric ratio of benzimidazole to zinc nitrate, a uniform ZIF-7 nanocrystals is formed at the surface of porous substrate. Then a regular and dense ZIF-7 membrane layer can be grown on the seeded substrate via secondary high-temperature crystallization. Fig. 6(c) and (d) depict the cross-section view of ZIF-7 membranes from single-step growth. It can be clearly found that the uneven ZIF-7 membrane layer is formed on the porous substrate. Note that the accumulation of ZIF-7 crystals on porous substrate is relatively loose compared with Fig. 6(a), which may result in some tiny defects in ZIF-7 membrane layer. Hence, two-step crystallization is more effective to prepare ZIF-7 membranes on porous substrate.

3-3. Permeation Flux and Ideal Selectivity of ZIF-7 Membranes

Table 1 and Fig. 8 depict single gas permeation properties of ZIF-7
Table 1. Permeation properties of the ZIF-7 membrane synthesized using two-step crystallization

\begin{tabular}{cccc}
\hline \hline Gas & $\begin{array}{c}\text { Permeance } \\
\left(10^{-7} \cdot \mathrm{mol} \cdot \mathrm{Pa}^{-1} \cdot \mathrm{s}^{-1}\right)\end{array}$ & $\begin{array}{c}\mathrm{H}_{2} \text { ideal selectivity } \\
\mathrm{P}_{i} / \mathrm{P}_{\mathrm{H} 2}\end{array}$ & $\begin{array}{c}\text { Knudsen constant } \\
{\left[\mathrm{M}_{i} / \mathrm{M}_{\mathrm{H} 2}\right]^{1 / 2}}\end{array}$ \\
\hline $\mathrm{H}_{2}$ & 25.15 & 1.00 & 1.00 \\
$\mathrm{CO}_{2}$ & 2.05 & 12.25 & 4.69 \\
$\mathrm{Ar}$ & 1.90 & 13.26 & 4.47 \\
$\mathrm{~N}_{2}$ & 1.87 & 13.43 & 3.74 \\
$\mathrm{CH}_{4}$ & 1.61 & 15.58 & 2.82 \\
\hline
\end{tabular}

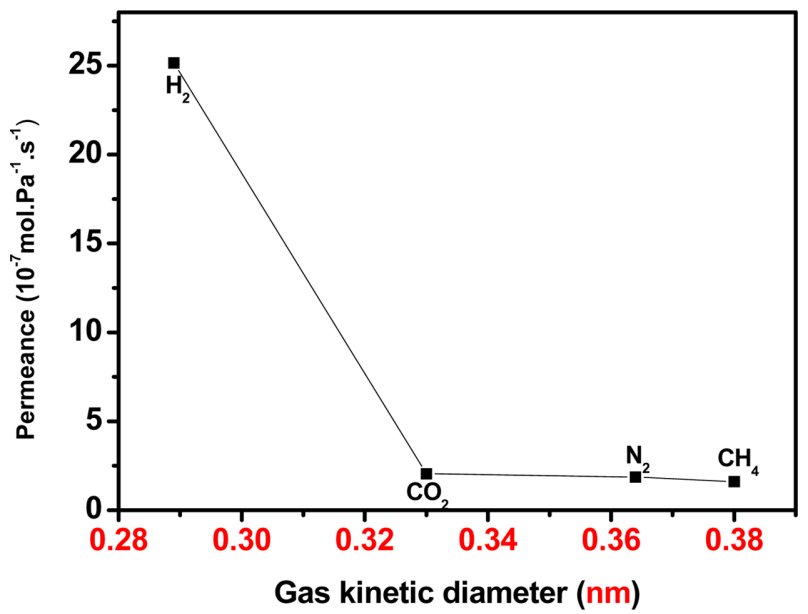

Fig. 8. Permeance of the ZIF-7 membrane from two-step crystallization versus the kinetic diameter of small molecular gases.

membrane from two-step crystallization. The whole test was finished on our homemade setup with $\mathrm{H}_{2}, \mathrm{CO}_{2}, \mathrm{Ar}, \mathrm{N}_{2}$ and $\mathrm{CH}_{4}$ using the Wicke-Kallenbach technique. The permeation data were calculated from volumetric flow rate at atmospheric pressure. It is shown that a much larger preferential permeance of $\mathrm{H}_{2}$ can be obtained with respect to other gases. The ideal separation factors of $\mathrm{H}_{2} / \mathrm{CO}_{2}, \mathrm{H}_{2} / \mathrm{Ar}$, $\mathrm{H}_{2} / \mathrm{N}_{2}$ and $\mathrm{H}_{2} / \mathrm{CH}_{4}$ are $12.25,13.26,13.43$ and 15.58 , respectively, which is much higher than their corresponding Knudson separation factor. The $\mathrm{H}_{2} / \mathrm{CO}_{2}$ ideal separation factor is close to the reported results of Li et al. [27], and the $\mathrm{H}_{2} / \mathrm{CH}_{4}$ ideal separation factor is higher than their reported results. It should be emphasized that our in-situ crystallization method is much simpler than their precoated seeding method. Thus, the ZIF-7 membrane prepared via two-step crystallization displays excellent molecular-sieving properties. Table 2 gives the permeation data of ZIF-7 membrane from single-step crystallization $\left(130^{\circ} \mathrm{C}\right.$ for $\left.24 \mathrm{~h}\right)$. It can be found that the ideal separation factors of $\mathrm{H}_{2} / \mathrm{CO}_{2}, \mathrm{H}_{2} / \mathrm{Ar}, \mathrm{H}_{2} / \mathrm{N}_{2}$ and $\mathrm{H}_{2} / \mathrm{CH}_{4}$ are all lower than

Table 2. Permeation properties of the ZIF-7 membrane synthesized using single step crystallization $\left(130^{\circ} \mathrm{C}\right.$ for $\left.24 \mathrm{hrs}\right)$

\begin{tabular}{cccc}
\hline \hline Gas & $\begin{array}{c}\text { Permeance } \\
\left(10^{-7} . \text { mol. Pa }^{-1} \cdot \mathrm{s}^{-1}\right)\end{array}$ & $\begin{array}{c}\mathrm{H}_{2} \text { ideal selectivity } \\
\mathrm{P}_{\mathrm{i}} / \mathrm{P}_{\mathrm{H} 2}\end{array}$ & $\begin{array}{c}\text { Knudsen constant } \\
{\left[\mathrm{M}_{\mathrm{i}} / \mathrm{M}_{\mathrm{H} 2}\right]^{1 / 2}}\end{array}$ \\
\hline $\mathrm{H}_{2}$ & 9.383 & 1.00 & 1.00 \\
$\mathrm{CO}_{2}$ & 3.113 & 3.01 & 4.69 \\
$\mathrm{CH}_{4}$ & 4.756 & 1.97 & 2.83 \\
$\mathrm{Ar}$ & 2.672 & 3.51 & 4.47 \\
$\mathrm{~N}_{2}$ & 3.206 & 2.93 & 3.74 \\
\hline
\end{tabular}




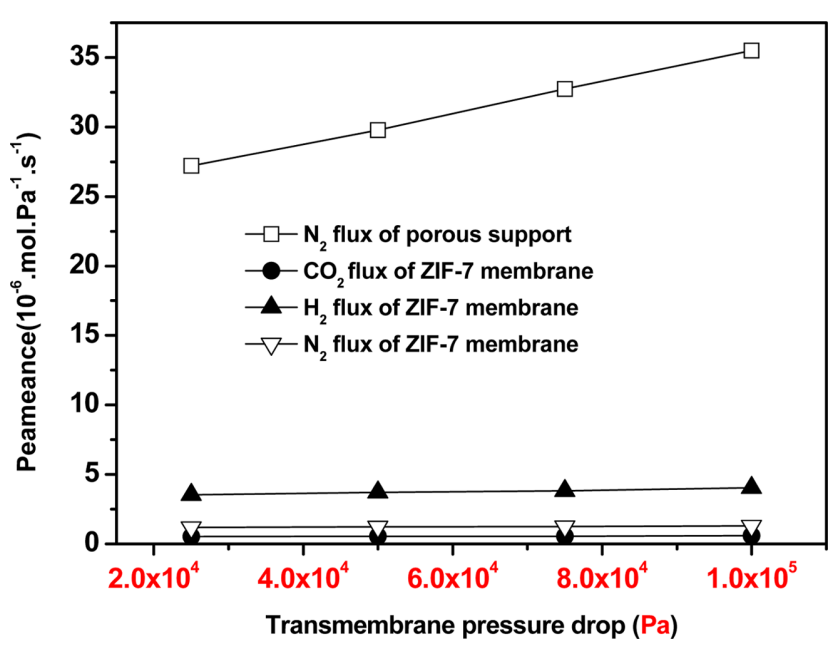

Fig. 9. A comparison of permeation flux through ZIF-7 membranes and porous substrate.

their corresponding Knudson separation factor, suggesting that there are some grain boundary defects in ZIF-7 membrane layer, which is consistent with the aforementioned discussion.

Permeation flux for $\alpha$-alumina porous substrate and ZIF-7 membrane from two-step crystallization is also given in Fig. 9. With increasing transmembrane pressure drop, $\mathrm{N}_{2}$ permeation flux through porous $\alpha$-alumina substrate increases remarkably, which indicates the presence of many macropore defects in $\alpha$-alumina substrate. For ZIF-7 membranes prepared by two-step crystallization, the permeation flux of $\mathrm{N}_{2}, \mathrm{H}_{2}$ and $\mathrm{CO}_{2}$ maintain relatively stable when increasing transmembrane pressure drop, which illustrates that no obvious macropore defects are present in the ZIF-7 membrane layer. As a result, the two-step crystallization method can produce a high-quality ZIF-7 membrane without obvious defects.

\section{Conclusions}

A two-step crystallization method was successfully developed to fabricate dense and defect-free ZIF-7 membrane on a-alumina porous substrate. ZIF-7 nanocrystals were deposited on porous substrate by in-situ room-temperature crystallization, and then, a high-temperature growth procedure was used to obtain dense ZIF-7 membrane layer. Compared with single-step crystallization, two-step crystallization can produce better ZIF-7 membrane. To produce ZIF-7 seeding nanocrystals, excessive benzimidazole was used to prepare the first precursor solution. Single gas permeation test proved that our prepared ZIF-7 membrane exhibits excellent molecule-sieving performance. The ideal separation factor of $\mathrm{H}_{2} / \mathrm{CO}_{2}, \mathrm{H}_{2} / \mathrm{Ar}, \mathrm{H}_{2} / \mathrm{N}_{2}$ and $\mathrm{H}_{2} / \mathrm{CH}_{4}$ are all higher than their corresponding Knudson separation factor.

\section{Acknowledgments}

This work was supported by National Natural Science Foundation (21201096) and the education department of Liaoning Province,
China (L2010242). The authors are also grateful for the financial support from the National Natural Science Foundation of China (No. 21103077), Program for New Century Excellent Talents in University (No. NCET-11-1011).

\section{References}

1. Tang, C. M. and Li, X. L., Korean J. Chem. Eng., 30(5), 169 (2012).

2. Han, S., Huang, Y. G., Watanabe, T., Nair, S., Walton, K. S., Sholl, D. S. and Meredith, J. C., Microporous Mesoporous Mater, 173, 86(2013).

3. Isaeva, V. I., Tkachenko, O. P., Brueva, T. R., Kapustin, G. I., Afonina, E.V., Mishin, I. V., Gryunert, V., Kustov, L. M., Solov'eva, S. E. and Antipin, I. S. Korean J. Chem. Eng., 85(4), 719(2011).

4. Song, J. L., Zhang, B. B., Jiang, T., Yang, G. Y. and Han, B. X., Korean J. Chem. Eng., 6(1), 21(2011).

5. Li, Q., Zhang, W., Miljanic, O. S., Knobler, C. B., Stoddart, J. F. and Yaghi, O. M., Chem. Commun., 46, 380(2010).

6. Volkringer, C. and Loiseau, T., Mater. Res. Bull., 41, 948 (2006).

7. Wu, H. B., Wei, S., Zhang, L., Xu, R., Hng, H. H., Lou, X. W., Chem. Eur. J., 19, 10804(2013).

8. Wu, X. F., Bao, Z. B., Yuan, B., Wang, J., Sun, Y. Q., Luo, H. M. and Deng, S. G., Microporous Mesoporous Mater., 180, 114 (2013).

9. Ebrahim, A. M., Levasseur, B. and Bandosz, T. J., Langmuir, 29, 168(2013).

10. Kong, X., Deng, H., Yan, F., Kim, J., Swisher, J. A., Smit, B., Yaghi, O. M. and Reimer, J. A., Science, 341, 882(2013).

11. Tao, K., Kong, C. L. and Chen, L., Chem. Eng. J., 220, 1(2013).

12. Moggach, S. A., Bennett, T. D. and Cheetham, A. K., Angew. Chem. Int. Ed., 48, 7087(2009).

13. Peralta, D., Chaplais, G., Paillaud, J. L., Simon-Masseron, A., Barthelet, K. and Pirngruber, G. D., Microporous Mesoporous Mater., 173, 1(2013).

14. Zheng, N., Park, K. S., Côte, A. P., Choi, J. Y., Huang, R. D., Uribe-Romo, F. J., Chae, H. K., O'Keeffe, M. and Yaghi, O. M., PNAS, 103, 10186(2006).

15. Huang, A., Bux, H., Steinbach, F. and Caro, J., Angew. Chem. Int. Ed., 49, 4958(2010).

16. Chizallet, C., Lazare, S., Bazer-Bachi, D., Bonnier, F., Lecocq, V., Soyer, E., Quoineaud, A. A. and Bats, N., J. Am. Chem. Soc., 132, 12365(2010).

17. Krishna, R. and van Baten, J. M., Sep. Purif. Technol., 87, 120 (2012).

18. Li, Y., Liu, J. and Yang, W., J. Membr. Sci., 281, 646(2006).

19. Lee, T., Choi, J. and Tsapatsis, M., J. Membr. Sci., 436, 79(2013).

20. Chmelik, C., van Baten, J. and Krishna, R., J. Membr. Sci., 397398, 87(2012).

21. Pan, Y., Wang, B. and Lai, Z., J. Membr. Sci., 421-422, 292 (2012).

22. Venna, S. R. and Carreon, M. A., J. Am. Chem. Soc., 132, 76(2009).

23. Bux, H., Liang, F., Li, Y., Cravillon, J., Wiebcke, M. and Caro, J., J. Am. Chem. Soc., 131, 1600(2009).

24. Hertäg, L., Bux, H., Caro, J., Chmelik, C., Remsungnen, T., Knauth, M. and Fritzsche, S., J. Membr. Sci., 377, 36(2011).

25. Liu, Y., Hu, E., Khan, E. A. and Lai, Z., J. Membr. Sci., 353, 36 
(2010).

26. Serre, C., Millange, F., Thouvenot, C., Noguès, M., Marsolier, G., Louër, D. and Férey, G., J. Am. Chem. Soc., 124, 13519(2002).

27. Li, Y., Liang, F., Bux, H., Yang, W. and Caro, J., J. Membr. Sci., 354, 48(2010).

28. Li, T., Pan, Y. C., Peinemann, K. V. and Lai, Z. P., J. Membr. Sci., 425-426, 235(2013).
29. Gücüyener, C., van den Bergh, J., Gascon, J. and Kapteijn, F., J. Am. Chem. Soc., 132, 17704(2010).

30. Nune, S. K., Thallapally, P. K., Dohnalkova, A., Wang, C., Liu, J. and Exarhos, G. J., Chem. Commun., 46, 4878(2010).

31. Shekhah, O., Wang, H., Zacher, D., Fischer, R. A. and Wöll, C., Angew. Chem. Int. Ed., 48, 5038(2009).

32. Ni, Z. and Masel, R. I., J. Am. Chem. Soc., 128, 12394(2006). 